Bull. Austral. Math. Soc.

VoL. 49 (1994) [21-24]

\title{
TWO COUNTER-EXAMPLES IN NONSEPARABLE BANACH SPACES
}

\section{G.A. Alexandrov and M.I. Kadec}

It is shown that the well-known theorem of Kadec for the $H_{\Gamma}$ renorming of separable Banach spaces, when $\Gamma$ is a norming subspace in the dual, cannot be extended to the class of nonseparable Banach spaces.

\section{INTRODUCTION}

Let $X$ be a Banach space and let $\Gamma$ be a total subspace in the dual space $X^{*}$.

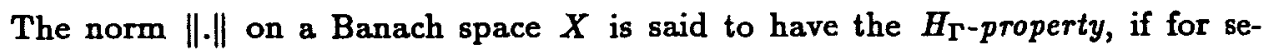
quences on the unit sphere, $\sigma(X, \Gamma)$ and norm convergence coincide, that is whenever $x_{0}, x_{n} \in X(n<\infty), \lim _{n}\left\|x_{n}\right\|=\left\|x_{0}\right\|$ and $\lim _{n} f\left(x_{n}\right)=f\left(x_{0}\right)$ for all $f \in \Gamma$, then $\lim _{n}\left\|x_{n}-x_{0}\right\|=0$.

The norm \|.\| on a Banach space $X$ is said to have the $K_{\Gamma}$-property, if the $\sigma(X, \Gamma)$ and norm topologies coincide on the unit sphere.

Obviously, if the norm has the $K_{\Gamma}$-property, then it has the $B_{\Gamma}$-property. The converse is not true.

When $\Gamma=X^{*}$, then the $H_{X} \cdot$-property is known as the $H$-property or the KadecKlee property and the $\boldsymbol{K}_{\boldsymbol{X}}$-property is known as the Kadec property.

If the Banach space $X$ admits an equivalent norm with the $H_{\Gamma}$ or $K_{\Gamma}$ property, then we write $X \in\left(H_{\Gamma}\right)$ or $X \in\left(K_{\Gamma}\right)$.

It is easy to see that, if $\Gamma$ is a total separable subspace in $X^{*}$, then $X \in\left(K_{\Gamma}\right)$ if and only if $X \in\left(H_{\Gamma}\right)$.

The following result of Kadec [5] is well-known: Let $X$ be a separable Banach space and $\Gamma$ is a norming subspace in $X^{*}$. Then $X \in\left(B_{\Gamma}\right)$.

Naturally, the question arises: Can we extend this result to the class of nonseparable Banach spaces?

The answer to this question is negative.

Plicko proved in [7] that, if $\Gamma$ is a total subspace in $X^{*}$ such that dens $(\Gamma)<$ dens $(X)$, then $X \notin\left(K_{\Gamma}\right)$. In particular, it follows that, if $X$ is a separable Banach space with a nonseparable dual space $X^{*}$, then $X^{*} \notin\left(H_{X}\right)$.

Received 20 January 1993

Copyright Clearance Centre, Inc. Serial-fee code: 0004-9729/94 \$A2.00+0.00. 
Here, we give two examples of total subspaces $\Gamma$ in $X^{*}$, for some concrete Banach spaces $X$, with dens $(\Gamma)=\operatorname{dens}(X)$, such that $X \notin\left(H_{\Gamma}\right)$.

We denote by $\overline{\operatorname{lin}}(A)$ the closed linear hull of the set $A \subset X$; $\operatorname{dens}(X)$ is the density character of $X$, that is, the smallest cardinal for which $X$ has a dense subset of the same cardinality.

Let $\Gamma$ be a subspace of $X^{*}$. We say that $\Gamma$ is norming if its Dixmier characteristic

$$
r(\Gamma)=\inf _{\|x\|=1} \sup _{f \in \Gamma} \frac{|f(x)|}{\|f\|}>0 .
$$

LEMMA. Let $\left(X, \rho_{1}\right)$ be an uncountable separable metric space, $\left(Y, \rho_{2}\right)$ be a separable metric space and $T: X \rightarrow Y$ be an arbitrary map. Then there exist point $x_{0} \in X$ and sequence $\left\{x_{n}\right\}_{n<\infty}$ in $X, x_{n} \neq x_{0}, \forall n<\infty$, such that $\lim _{n} \rho_{1}\left(x_{n}, x_{0}\right)=0$ and $\lim _{n} \rho_{2}\left(T x_{n}, T x_{0}\right)=0$.

In this case, we say that $x_{0}$ is a point of partial continuity for the map $T$.

\section{First eXample}

Let $A P$ be the Banach space of all almost periodic functions defined on the real line $\mathbb{R}$ with the supremum norm $\|\cdot\|_{\infty}$.

We define the linear functionals $\delta_{t} \in A P^{*}$ for every $t \in \mathbb{R}$ by the equality $\delta_{t}(f)=$ $f(t), f \in A P$, and define the subspace $\Gamma=\varlimsup_{\ln }\left(\delta_{t}\right)_{t \in \mathbb{R}}$ in $A P^{*}$.

The subspace $\Gamma$ is norming.

Really, if $f \in A P,\|f\|_{\infty}=1$, then there exists a sequence $\left\{t_{n}\right\}_{n<\infty} \subset R$ such that $\lim _{n}\left|f\left(t_{n}\right)\right|=1$, that is $\lim _{n}\left|\delta_{t_{n}}(f)\right|=1$. Since $\sup _{\delta \in \Gamma}(|\delta(f)| /\|\delta\|) \geqslant\left|\delta_{t_{n}}(f)\right|, \forall n<\infty$, then $r(\Gamma)=1$.

Proposition 1. The space $A P \notin\left(H_{\Gamma}\right)$.

Proof: Let $f_{\lambda}(t)=e^{i \lambda t}, \lambda \in \mathbb{R}$, and let $\|$.$\| be an equivalent norm on Banach$ space $A P$.

We examine the function $\lambda \mapsto\left\|f_{\lambda}\right\|, \lambda \in \mathbb{R}$.

According to the Lemma, there exists a point of partial continuity for this function, that is, there exist $\lambda_{0}, \lambda_{n} \in \mathbb{R}, \lambda_{n} \neq \lambda_{0}(n<\infty)$ such that

$$
\begin{aligned}
\lim _{n} \lambda_{n} & =\lambda_{0}, \\
\lim _{n}\left\|f_{\lambda_{n}}\right\| & =\left\|f_{\lambda_{0}}\right\| .
\end{aligned}
$$

From (1) and the definition of the functions $f_{\lambda_{n}}$ we get $\lim _{n} f_{\lambda_{n}}(t)=f_{\lambda_{0}}(t), \forall t \in \mathbb{R}$, which is equivalent to

$$
\lim _{n} \delta_{t}\left(f_{\lambda_{n}}\right)=\delta_{t}\left(f_{\lambda_{0}}\right), \quad \forall t \in \mathbb{R}
$$


Consequently, from (3) for all $\delta \in \Gamma$ we have

$$
\lim _{n} \delta\left(f_{\lambda_{n}}\right)=\delta\left(f_{\lambda_{0}}\right) \text {. }
$$

Now, if we suppose that the space $A P \in\left(H_{\Gamma}\right)$ then from (2) and (4) it follows that $\lim _{n}\left\|f_{\lambda_{n}}-f_{\lambda_{0}}\right\|=0$ which is, obviously, impossible, since the system $\left\{f_{\lambda}\right\}_{\lambda \in \mathbb{R}}$ is minimal (see [6]). The proposition is proved.

\section{SECOND EXAMPLE}

Let $Q C[0,1]$ be the Banach space of all real-valued functions defined on $[0,1]$ for which $f(t+0)=f(t)$ for every $t$, that is, $f$ is continuous from the right and $f(t-0)$ exists for every $t$ with the supremum norm $\|\cdot\|_{\infty}$.

Let $E$ be a dense subset in $[0,1]$ such that the set $E_{1}=[0,1] \backslash E$ is uncountable.

We define the linear functionals $\delta_{t} \in Q C[0,1]^{*}$ for every $t \in E$ by the equality $\delta_{t}(f)=f(t), f \in Q C[0,1]$, and define the subspace $\Gamma=\overline{\ln }\left(\delta_{t}\right)_{t \in E}$ in $Q C[0,1]^{*}$.

The subspace $\Gamma$ is norming.

Really, if $f \in Q C[0,1],\|f\|_{\infty}=1$, then there exists a sequence $\left\{t_{n}\right\}_{n<\infty} \subset E$ such that $\lim _{n}\left|f\left(t_{n}\right)\right|=1$, that is, $\lim _{n}\left|\delta_{t_{n}}(f)\right|=1$. Since $\sup _{\delta \in \Gamma}(|\delta(f)| /\|\delta\|) \geqslant\left|\delta_{t_{n}}(f)\right|$, $\forall n<\infty$, then $r(\Gamma)=1$.

Proposition 2. The space $Q C[0,1] \notin\left(H_{\Gamma}\right)$.

Proof: For every $s \in E_{1}$ we define the functions $f_{0}(t)=0$, if $0 \leqslant t<s$ and $f_{s}(t)=1$, if $s \leqslant t \leqslant 1$. Obviously, $f_{s} \in Q C[0,1]$ for all $s \in E_{1}$. Let $\|\cdot\|$ be an equivalent norm on the Banach space $Q C[0,1]$.

We examine the function $s \mapsto\left\|f_{s}\right\|, s \in E_{1}$.

According to the Lemma, there exists a point of partial continuity for this function, that is, there exist $s_{0}, s_{n} \in E_{1}, s_{n} \neq s_{0}(n<\infty)$ such that

$$
\begin{aligned}
\lim _{n} s_{n} & =s_{0}, \\
\lim _{n}\left\|f_{e_{n}}\right\| & =\left\|f_{s_{0}}\right\| .
\end{aligned}
$$

From (5) and definition of the functions $f_{s_{n}}$ we get $\lim _{n} f_{s_{n}}(t)=f_{a_{0}}(t), \forall t \in E$, which is equivalent to

$$
\lim _{n} \delta_{t}\left(f_{e_{n}}\right)=\delta_{t}\left(f_{\Delta_{0}}\right), \quad \forall t \in E .
$$

Consequently, from (3) for all $\delta \in \Gamma$ we have

$$
\lim _{n} \delta\left(f_{e_{n}}\right)=\delta\left(f_{s_{0}}\right) \text {. }
$$

Now, if we suppose that the space $Q C[0,1] \in\left(B_{\Gamma}\right)$ then from (6) and (8) it follows that $\lim _{n}\left\|f_{o_{n}}-f_{s_{0}}\right\|=0$ which is, obviously, impossible, since $\left\|f_{s_{n}}-f_{\iota_{0}}\right\|=1$ for all $n<\infty$. The proposition is proved. 
REMARKs. (i) The spaces $A P$ and $Q C[0,1]$ possess an equivalent locally uniformly convex norm and, consequently, they have the Kadec property (see $[1,2,3]$ ).

(ii) Godun proved in [4] that, if $\left(x_{i}, f_{i}\right)_{i \in I}$ is $M$-basis in the Banach space $X$ and $\Gamma=\overline{l i n}\left(f_{i}\right)_{i \in I}$, then $X \in\left(H_{\Gamma}\right)$ if and only if the subspace $\Gamma$ is norming.

This gives rise to the following.

QUESTION. Let $X$ be a nonseparable Banach space and let $\Gamma$ be a norming subspace in dual the space $X^{*}$. What sufficient conditions must $\Gamma$ satisfy so that $X$ admits the $H_{\Gamma}$-property?

\section{References}

[1] G.A. Alexandrov, Locally uniformly convex equivalent norms in nonseparable Banach spaces, (in Russian), Ph.D. Dissertation: Kharkov, 1980.

[2] G.A. Alexandrov and K.-D. Kursten, 'Some nonseparable Banach spaces which admit equivalent locally uniformly convex norms', in Russian, Izv. Vyssh. Ucheben. Zaved. Mat. 7 (1981), 10.

[3] G.A. Alexandrov, 'Equivalent locally uniformly convex renorming of nonseparable Banach spaces', in Russian, in Constructive function theory 81 (Varna 1981) (Bulgar. Acad. Sci., Sofia, 1983), pp. 9-13.

[4] B.V. Godun, 'On the Markushevich bases', in Russian, Dokl. Acad. Nauk SSSR 266 (1982), 11-14.

[5] M.I. Kadec, 'On the connection between weak and strong convergence', in Ukraninian, Dopovidi Acad. Nauk Ukrain. RSR 9 (1957), 949-952.

[6] L.H. Loomis, An introduction to abstract harmonic analysis (D. van Nostrand Co, Toronto, New York, London, 1953).

[7] A.N. Plicko, 'On the projective resolution of identity, the Markushevich bases and the equivalent norms', in Russian, Mat. Zametki 34 (1983), 719-726.

Department of Mathematics and Informatics

University of Sofia

5 J. Bourchier Blvd

1126 Sofia

Bulgaria
Pravda prosp. 5

Flat 26

310022-Kharkov

Ukraine 